\title{
On the classes of Lipschitz and smooth conjugacies of unimodal maps
}

\author{
by
}

Waldemar Pałuba (Warszawa)

\begin{abstract}
Under very mild assumptions, any Lipschitz continuous conjugacy between the closures of the postcritical sets of two $C^{1}$-unimodal maps has a derivative at the critical point, and also on a dense set of its preimages. In a more restrictive situation of infinitely renormalizable maps of bounded combinatorial type the Lipschitz condition automatically implies the $C^{1}$-smoothness of the conjugacy. Here the critical degree can be any real number $\alpha>1$.
\end{abstract}

1. Introduction. This work is devoted to the study of properties of conjugacies of some unimodal maps of an interval. Those properties have for long attracted interest of researchers in the field of nonlinear science. Much progress has been made here over the past two decades, mostly due to the joint employment of both complex and real techniques. However, those powerful techniques seem to be intrinsically limited to the maps which have singularities of an integer critical degree. This is more than a mere inconvenience. For noninteger critical degrees $\alpha>1$ there is often strong numerical evidence for essentially similar patterns of behavior, for which we lack any "pure thought" explanation. A survey of classical (up to the early 1990's) results can be found in the monographs of P. Collet and J. P. Eckmann [2] or W. de Melo and S. van Strien [5]. Among the valuable but few results that prove a generalized version of a property formerly known for the critical degree $\alpha=2$ there is a recent work of W. Shen (see [9]). The interest in a real-variable approach to those problems seems to be reviving. We intend to contribute to this research in a series of three papers. The current article is the first of them and covers the most general results about Lipschitz continuous conjugacies. The following one is [7].

Let us consider a pair of topologically conjugate one-dimensional maps. We would like to know as much as possible what one can expect of the conjugating homeomorphism in terms of Hölder class, symmetry, smoothness an

2000 Mathematics Subject Classification: Primary 37C15. 
so forth, provided the initial systems are smooth enough. Some properties, like quasisymmetry for instance, are sought for on the whole domain. Smooth conjugacy results are usually a bit more subtle and sometimes require extra assumptions. First, there can appear obvious obstacles to smoothness, like for instance multipliers of the periodic orbits in the case of diffeomorphisms of the circle (or renormalizable maps of an interval, see Pałuba [6]). Even in the more interesting situation when there are no obstacles of that kind, the conjugacy tends to be, in general, less differentiable than the mappings. This problem was studied for more than half of the past century (cf. Finzi [3]), and there are renown results of Arnold [1], Herman [4], Yoccoz [12] and others on conditions for smoothness of the conjugacies.

In another, very important setting, the presence of hyperbolic expansion in the system is essential (e.g. Sullivan and Shub [10]).

For nonhyperbolic unimodal maps of an interval this problem is also typical: there are obstacles to smoothness of the conjugacy on the whole domain and even for analytic maps restricted to invariant subdomains their conjugacies cannot be, in general, expected to be in any better class of smoothness than $C^{1+\epsilon}$, where $\epsilon$ depends on the geometry of the postcritical orbit (cf. Rand [8], or Pałuba [6]). Thus, in the case of maps of an interval, the closure of the postcritical set is the natural choice of an invariant subdomain where we look for possible smoothness of the conjugacy. From now on, we shall be restricting our maps to these subdomains and study their conjugacy $h$ there.

First, in Section 2, we give a nice and rather surprising result: if $h$ is Lipschitz continuous on the restricted domain, then it must automatically be differentiable at the critical point. No extra assumptions on the dynamics of our system other than the recurrence of the critical point are needed for this statement to hold (except that in the setting of $C^{1}$-smoothness we also have to get rid of some immediate obstacles, like wandering intervals). The essence of the argument we give in Section 2 is the repeated use of homogeneity of the power-law map $x \mapsto|x|^{\alpha}$.

The presence of some extra combinatorial conditions that make the geometry of the restricted domains simple enough allows for a further strengthening of our result. In Section 3 we use the well understood structure of the infinitely renormalizable maps of bounded combinatorial type (see [11] and [5]) to derive (Corollary 1 of Theorem 2) that for those maps the classes of Lipschitz continuous conjugacies on the restricted domains coincide with the classes of $C^{1}$-smooth conjugacies. This comes as a rather technical consequence of Theorem 1 of Section 2 and the so called bounded geometry property of our restricted domains.

The results of Section 3 may seem to resemble the well-known statements about Lipschitz continuous or $C^{1+\epsilon}$-smooth conjugacies on the in- 
variant Cantor sets (cf. [8], [6] and the last chapter in [5]). However, the latter results were derived from the exponential contracting property of the renormalization operator. Here, we want to emphasize very strongly that no convergence - not even nonexponential! - of renormalization needs to be assumed for Theorem 2 to hold. What we actually prove is that as soon as we know about the Lipschitz continuity of $h$ we can show it is $C^{1}$-smooth; then, convergence of renormalization (though not an exponential convergence) can be derived from the Lipschitz condition itself. So what we have here is a purely qualitative statement: the problem of universality of the Feigenbaum-like attracting Cantor sets reduces to the question of Lipschitz continuity of the conjugacies.

It is generally expected that for the maps we study in Section 3 the derivative of $h$ should be Hölder continuous $\left(C^{1+\epsilon}\right.$-smoothness of $h$ ), and of course the very presumption of Lipschitz continuity of $h$ should be redundant. While the bounds on the geometry of the Cantor sets we deal with do provide for the quasisymmetry of $h$, we do not intend to suggest that closing the gap between the quasisymmetry and the Lipschitz continuity of $h$ could possibly be easy (but then we would be getting the universality of the Feigenbaum attractors also for the noninteger critical degrees). Thus, what we get here is a global result for all $\alpha>1$ at the cost of stronger starting hypothesis.

Finally, we outline the logical structure of the proofs in this work. We start with the conjugacy between postcritical orbits of the two maps and introduce a notion of distortion of the conjugacy itself from being smooth. The power-law singularity makes this distortion decrease every time the itinerary passes through a small enough vicinity of the critical point $c$. Since we are close to $c$ infinitely many times, we conclude that, in the infinitesimally small scale, the distortion is nonexistent and so the conjugacy is differentiable (Theorem 2 and the subsequent Corollary). Technically the argument turns out more complicated, but the underlying idea is exactly as described. We emphasize that throughout we work with a pair of maps (and their conjugacy) rather than with the intrinsic properties shared by all the maps in the class considered. While the original argument can be carried out also in the setting of Hölder bounds, the difficulty is that only with Lipschitz bounds in place is it true that what we get from this argument is more than we put into it.

Why the Lipschitz bounds should hold for all singularities $\alpha>1$ remains unknown.

2. Upgrading Lipschitz continuous conjugacies. In this section, we shall be concerned with dynamical systems arising from $C^{1}$-smooth unimodal maps of the interval $I$ with singularity of the critical order $\alpha>1$ at 
the critical point $c$. Here $\alpha$ is an arbitrary real number greater than 1 , not necessarily an integer.

For simplicity of notation we assume that $I$ is an interval symmetric about $0(c=0)$ and $f: I \rightarrow I$ is a map of the type $f=\phi\left(|x|^{\alpha}\right)$, where $\phi$ is a $C^{1}$-smooth diffeomorphism with range within $I$.

We fix the critical degree $\alpha$ and let $\mathcal{A}$ denote the class of maps $f$ of the above type, such that:

- the critical point of $f$ is recurrent,

- the postcritical set $\mathcal{C}_{f}=\operatorname{cl}\left\{f^{n}(c)\right\}_{n=1}^{\infty}$ contains a dense subset made of some preimages of $c$.

The latter condition has been explicitly stated to avoid possible silly pathologies (i.e. wandering intervals) that can arise when we deal with systems which are only $C^{1}$-smooth.

$\mathcal{C}_{f}$ is a closed metric subspace of $I$. The action of $f$ restricted to $\mathcal{C}_{f}$, $\tilde{f}=\left.f\right|_{\mathcal{C}_{f}}$, makes the pair $\left\{\tilde{f}, \mathcal{C}_{f}\right\}$ into a transitive topological dynamical system. From now on we restrict our attention to this system.

Let us consider another map in the class $\mathcal{A}$, say $g$, and suppose that the systems $\left\{\widetilde{f}, \mathcal{C}_{f}\right\},\left\{\widetilde{g}, \mathcal{C}_{g}\right\}$ are topologically conjugate by a homeomorphism $h: \mathcal{C}_{f} \rightarrow \mathcal{C}_{g}$ (in this section we shall not need to extend the homeomorphism $h$ to the whole interval). Set

$$
\bar{x}:=h(x) .
$$

It makes sense to say that $h$ has a derivative at a point $x$ in $\mathcal{C}_{f}$ if the limit

$$
\lim _{y \rightarrow x, y \in \mathcal{C}_{f}} \frac{\bar{y}-\bar{x}}{y-x}
$$

exists. Here we have a theorem.

THeOREm 1. If the maps $f$ and $g$ are both in the class $\mathcal{A}$ and the generated systems $\left\{\tilde{f}, \mathcal{C}_{f}\right\},\left\{\widetilde{g}, \mathcal{C}_{g}\right\}$ are topologically conjugate by a homeomorphism $h$ such that $h$ and $h^{-1}$ are both Lipschitz continuous, then there exist nonvanishing derivatives $h^{\prime}(c)$ and $\left(h^{-1}\right)^{\prime}(h(c))$. Moreover, the first derivatives of $h\left(\right.$ and of $\left.h^{-1}\right)$ exist at all preimages of the critical point $c$ contained in $\mathcal{C}_{f}$ (in $\mathcal{C}_{g}$ respectively).

Proof. We may assume that all the points in the following considerations are actually preimages of $c$, since those are dense in $\mathcal{C}_{f}$. It will become clear throughout the proof that this assumption does not cause any loss of generality. This becomes relevant in Step 2 of the proof.

For all points $t \in \mathcal{C}_{f}$, we will introduce two quantities which locally measure the "distortion from smoothness" (or more precisely: "distortion from symmetry", but here we work under the hypothesis of Lipschitz condition). They will be referred to as badness and (capitalized) Badness. Here they 
are:

$$
\text { badness }=b(t)=\underset{\delta \rightarrow 0}{\limsup } b_{\delta}(t)
$$

where $b_{\delta}(t)$ is given by

$$
b_{\delta}(t)=\left(\sup _{z \in(x-\delta, x+\delta) \cap \mathcal{C}_{f}}\left|\frac{t-z}{\bar{t}-\bar{z}}\right|\right):\left(\inf _{z \in(x-\delta, x+\delta) \cap \mathcal{C}_{f}}\left|\frac{t-z}{\bar{t}-\bar{z}}\right|\right) .
$$

Similarly,

$$
\text { Badness }=B(t)=\underset{\delta \rightarrow 0}{\limsup _{\delta \rightarrow 0}} B_{\delta}(t)
$$

where

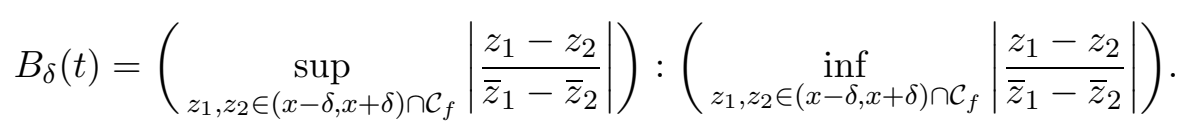

Of course we always have $M / K \geq B \geq b \geq 1$, where $M$ and $K$ are the upper and lower Lipschitz bounds on $h$ respectively. Notice that by their very definition Badness (and badness) are $C^{1}$-smoothly invariant.

Differentiability of $h$ at $c$ amounts to $b(c)=1$, since we have the Lipschitz condition in hand.

We assume that $c$ is not periodic; otherwise the theorem holds trivially.

Proceeding by contradiction, suppose that the derivative does not exist; then there is a positive $\varepsilon$ such that arbitrarily close to $c$ one can find a pair of points $x, y \in \mathcal{C}_{f}$ satisfying

$$
\frac{\bar{y}}{y}: \frac{\bar{x}}{x}>1+\varepsilon
$$

In the argument below we proceed along the following lines. Assuming that badness is not equal to 1, we consider the (capitalized) Badness. Making essential use of the homogeneity of the power-law map, combined with the lower bound on badness, we prove that there exists an infinite sequence of close returns into nested neighborhoods of the critical point, such that in each of those returns the a priori bounds on Badness are getting strictly closer to 1 .

Eventually, by the $C^{1}$-invariance of Badness we derive that Badness must have been equal to 1 from the beginning, which contradicts the hypothesis we made about the badness.

STEP 1: The "four points" argument. We find $x, y$ satisfying (1) in $U$, where $U$ is some small neighborhood of 0 . On tiny neighborhoods of $x$ and $y$ the mapping $f$ acts almost linearly, as $f^{\prime}(x)$ and $f^{\prime}(y)$ respectively, with an arbitrarily small error. On the conjugate picture $g$ acts almost linearly in small (conjugate) neighborhoods of $\bar{x}$ and $\bar{y}$. Thus by $C^{1}$-invariance the 
following estimates hold:

$$
B(f(x))=B(x) \leq \frac{M}{K} e^{-\left|\log \frac{f^{\prime}(x)}{g^{\prime}(\bar{x})}\right|}
$$

and also

$$
B(f(y))=B(y) \leq \frac{M}{K} e^{-\left|\log \frac{f^{\prime}(y)}{g^{\prime}(\bar{y})}\right|} .
$$

The maps $f$ and $g$ are both of the type power-law postcomposed with a diffeomorphism, say $f=\phi\left(|x|^{\alpha}\right)$ and $g=\psi\left(|x|^{\alpha}\right)$. If the neighborhood $U$ has been chosen small enough, then the term in the chain rule for the derivative, coming from the diffeomorphism part, is about the same for both $x$ and $y$, and approximately equals $\phi^{\prime}(0)$ (or $\psi^{\prime}(0)$ respectively for $\left.|\bar{x}|,|\bar{y}|\right)$. Now the key argument for the discrepancy between the values of the logarithms in (2) and (3) comes from the homogeneous power-law part.

For a homogeneous map the ratio of the arguments determines the ratio of the corresponding derivatives. Therefore whenever $\bar{y} / \bar{x}$ differs "significantly" from $y / x$ (as in (1)), so does the quantity $f^{\prime}(\bar{y}) / f^{\prime}(\bar{x})$ compared to $f^{\prime}(y) / f^{\prime}(x)$. Precisely, taking into account that the derivative of the powerlaw map is of the form $\alpha \cdot|x|^{\alpha-1}$, the inequality (1) yields

$$
\frac{f^{\prime}(y)}{g^{\prime}(\bar{y})}<\frac{f^{\prime}(x)}{g^{\prime}(\bar{x})} \cdot \frac{1}{(1+\varepsilon)^{\alpha-1}}
$$

provided that the neighborhood $U$ was small enough.

Consequently, at least one of the absolute values of the logarithms in (2) or (3), say the one in (2), has to be larger than $-\log \beta$, where

$$
-\log \beta=\frac{\alpha-1}{2} \log (1+\varepsilon) .
$$

Thus we get

$$
B(x)<\frac{M}{K} \beta .
$$

So we have found a point, namely $x$, at which Badness does not reach the a priori upper bound of $M / K$ but is smaller than $M / K$ by a definite multiplicative factor of $\beta<1$.

(4) also holds true for $\delta$-Badness $B_{\delta}$ if $\delta$ is small enough. Once again, replacing the stretching (or contraction) of the lengths of all short intervals in a sufficiently small neighborhood of $x\left(\bar{x}\right.$, respectively) by $\left|f^{\prime}(x)\right|$ (or $\left|g^{\prime}(\bar{x})\right|$, respectively), we see that $\delta$-Badness, and so Badness itself, must be bounded from above by $\beta M / K$ not only at $x$, but also at all points of $\mathcal{C}_{f}$ sufficiently close to $x$.

STEP 2: Recurrence. Now, recall that $x$ (and $y$ ) could have been chosen as a preimage of $c$, say $f^{m}(x)=c$. By the smooth invariance of Badness, $B\left(f^{l}(x)\right)=B(x)$ for any finite $l$ so $B(c)<\beta M / K$. By the way, notice 
that due to this remark, Badness must also be smaller than $\beta M / K$ at any preimage of $c \in \mathcal{C}_{f}$.

On tiny neighborhoods of $x$ the action of $f^{m}$ is virtually linear with arbitrarily small error, so $\delta$-Badness can be transported forward finitely many steps nearly unperturbed, if $\delta$ is small enough. Thus we also have $B_{\delta_{1}}(c)<\beta M / K$, provided $\delta_{1}$ is sufficiently small. Put $U_{0}=U$ and let $U_{1}$ be a symmetric neighborhood with diameter equal to such a $\delta_{1}$. The same can be claimed (upon possibly decreasing $\delta_{1}$ further) for all points $t \in \mathcal{C}_{f} \cap U_{1}$ : Badness at those points satisfies $B(t)<\beta M / K$, and this same inequality holds for $\delta$-Badness with sufficiently small $\delta$. Let $l$ be the time of first return of the orbit of $c$ into such a neighborhood $U_{1}$.

Now consider a new $x$ and a new $y$ (we keep the same notation as in Step 1) which satisfy (1); additionally we require them to be very close to $c$, compared to the diameter of $U_{1}$. On the neighborhoods of those new $x$ and $y$ with tiny diameter as compared to $\min (|x|,|y|), f$ acts almost linearly changing the lengths of intervals by approximately $\left|f^{\prime}\right|$. On the conjugate picture the same is true for $g$.

At the first iterate of $f$ (and $g$ ), we apply a homogeneous map postcomposed with a diffeomorphism, so there is a definite discrepancy between the ratios of the derivatives for $f$ and $g$, as in Step 1. Then along the orbits of $f(x)$ and $f(y)$ the mapping $f^{l-1}$ acts almost linearly on the intervals $f$ (tiny neighborhood of $x$ ) and $f$ (tiny neighborhood of $y$ ) respectively, and similarly for $g$. However, this time we return into $U_{1}$ (and $\bar{U}_{1}$ ), where we knew a priori that Badness is bounded by $\beta M / K$, so we have a modified version of (2), (3), namely

$$
\begin{aligned}
& B\left(f^{l}(x)\right)=B(x) \leq \beta \frac{M}{K} e^{-\mid \log \frac{f^{\prime}(x)}{g^{\prime}(\bar{x})} \cdot \frac{\left(f^{(l-1)}\right)^{\prime}(f(x))}{\left(g^{(l-1)}\right)^{\prime}(g(\bar{x}))}}, \\
& B\left(f^{l}(y)\right)=B(y) \leq \beta \frac{M}{K} e^{-\mid \log \frac{f^{\prime}(y)}{g^{\prime}(\bar{y})} \cdot \frac{\left(f^{(l-1)}\right)^{\prime}(f(y))}{\left(g^{(l-1)}\right)^{\prime}(g(\bar{y}))}} .
\end{aligned}
$$

Now notice that choosing the new $x$ and $y$ sufficiently close to $c$ we can have the distance $|f(x)-f(y)|(|g(\bar{x})-g(\bar{y})|$, respectively) so small that $\left(f^{(l-1)}\right)^{\prime}(f(x)) /\left(f^{(l-1)}\right)^{\prime}(f(y))$ and $\left(g^{(l-1)}\right)^{\prime}(g(\bar{x})) /\left(g^{(l-1)}\right)^{\prime}(g(\bar{y}))$ will be both arbitrarily close to 1 , or in other words the quantities

$$
\frac{\left(f^{(l-1)}\right)^{\prime}(f(x))}{\left(g^{(l-1)}\right)^{\prime}(g(\bar{x}))}, \quad \frac{\left(f^{(l-1)}\right)^{\prime}(f(y))}{\left(g^{(l-1)}\right)^{\prime}(g(\bar{y}))}
$$

can be made nearly identical, with an arbitrarily small error.

Thus, by the argument as in Step 1, at least one of the absolute values of the logarithms in (5) or (6) has to be larger than $-\log \beta$. This tells us that $B(x)$ (and/or equivalently $B(y)$ ) does not exceed $\beta^{2} M / K$. Neither does $B(c)$, as $x$ is a preimage of $c$. So also $\delta$-Badness, for sufficiently small 
$\delta$ 's on an appropriately small symmetric neighborhood $U_{2}$ of $c$, is smaller than $\beta^{2} M / K$, by the same argument we used for $U_{1}$ at the beginning of this recurrence step.

Applying this argument recurrently to a nested sequence of neighborhoods $U_{2}, U_{3}$, etc. $[\log (K / M) / \log \beta]+1$ times, we get $B(c)<1$, a contradiction.

Similarly, because of the Lipschitz bounds, the derivative $\left(h^{-1}\right)^{\prime}(h(c))$ also exists. Of course for all positive integers $i$ and all inverse branches such that $f^{-i}(c) \in \mathcal{C}_{f}$ we also have $b\left(f^{-i}(c)\right)=1$ and so the derivative of $h$ exists at all the preimages of $c$ in $\mathcal{C}_{f}$.

REMARK 1. In our general setting there is no way to carry this differentiability property over onto the whole set $\mathcal{C}_{f}$, despite the density of the preimages of $c$. Compare the next section.

REMARK 2. Notice that although Badness rather than badness was our main tool in the proof we cannot prove in this general setting that $B(c)=1$. Actually we have only proved that $b(c)$ must be equal to 1 , because otherwise $B(c)=1$. But if $b(c)=1$, there is no contradiction in assuming $B(c)>1$.

3. The infinitely renormalizable case. In this section we restrict our attention to those maps in $\mathcal{A}$ that are infinitely renormalizable of bounded combinatorial type. We also require them to be $C^{2}$-smooth, except of course in the immediate vicinity of the critical point, where the power-law map $x \mapsto|x|^{\alpha}$ is not $C^{2}$ if $\alpha<2$.

The maps in the class $\mathcal{A}$ that are of the form $f=\phi\left(|x|^{\alpha}\right)$ with the diffeomorphism $\phi$ being of class $C^{2}$ rather than $C^{1}$ is a subclass of $\mathcal{A}$ which we shall simply denote by $\mathcal{A}^{2}$.

Our main goal is to investigate the classes of Lipschitz continuous conjugacies for maps like this. The conjugating homeomorphism $h$ can now be defined on the whole interval, $h: I \rightarrow I$. The Lipschitz continuity of such an $h$ requires an additional condition on the eigenvalues of the periodic orbits of $f$ and $g$ (see [6]). So in general the class of Lipschitz conjugacy (on the whole $I$ ) is small. Thus we restrict $h$ to the closure of the postcritical orbit $\mathcal{C}_{f}$ as in the previous section. We will be assuming that the restricted conjugating homeomorphism $h: \mathcal{C}_{f} \rightarrow \mathcal{C}_{g}$ satisfies the bi-Lipschitz condition with upper and lower bounds $M$ and $K$ respectively. From now on we consider a pair of conjugate maps $f$ and $g$ satisfying all of the hypotheses above.

Let $L_{n} \ni c, n=0,1,2, \ldots$, be the basic small renormalization interval (i.e. bounded by the appropriate points of the postcritical orbit) at depth $n$ in the process of renormalization, $L_{0}=\left[f(c), f^{2}(c)\right]$.

By the definition of $L_{n}$ there exists a positive integer $r=r(f, n)$ such that $f^{r} L_{n}=L_{n}$ and $f^{i} L_{n} \cap f^{j} L_{n}=\emptyset$ for $0 \leq i<j<r$. Denote by $C_{n}$ the 
collection of intervals $\left\{f^{i} L_{n}\right\}_{i=0}^{r-1}$. Of course

$$
\mathcal{C}_{f}=\bigcap_{n \geq 0} \bigcup_{\mathcal{L} \in C_{n}} \mathcal{L}
$$

Bounded combinatorics with bound $T$ means that at most $T$ elements of $C_{n+1}$ are contained in $L_{n}$ for all $n \geq 0$.

Now in place of standard Lipschitz bounds $K$ and $M$ on $\left.h\right|_{\mathcal{C}_{f}}$ we shall consider new bounds $\mathcal{K}, \mathcal{M}$ related to the way the Cantor set $\mathcal{C}_{f}$ is obtained through the nested family of collections of intervals. Set

$$
\mathcal{K}_{n}=\inf _{\mathcal{L} \in \bigcup_{i \geq n}} \frac{|\mathcal{L}|}{|\overline{\mathcal{L}}|}, \quad \mathcal{M}_{n}=\sup _{\mathcal{L} \in \bigcup_{i \geq n}} \frac{|\mathcal{L}|}{|\overline{\mathcal{L}}|}
$$

and

$$
\mathcal{K}=\lim _{n \rightarrow \infty} \mathcal{K}_{n}, \quad \mathcal{M}=\lim _{n \rightarrow \infty} \mathcal{M}_{n} .
$$

Clearly $K \leq \mathcal{K} \leq h^{\prime}(c) \leq \mathcal{M} \leq M$, where $h^{\prime}(c)$ is the derivative of the conjugacy at the critical point $c$ in the sense of the previous section.

As a technical tool we will introduce one more notion of distortion. For $x \in \mathcal{C}_{f}$ set

$$
\begin{gathered}
\mathcal{B}_{\delta}(x)=\left(\sup _{\mathcal{L} \subset(x-\delta, x+\delta), \mathcal{L} \in \cup C_{i}} \frac{|\mathcal{L}|}{|\overline{\mathcal{L}}|}\right):\left(\inf _{\mathcal{L} \subset(x-\delta, x+\delta), \mathcal{L} \in \cup C_{i}} \frac{|\mathcal{L}|}{|\overline{\mathcal{L}}|}\right), \\
\mathcal{B}(x)=\lim _{\delta \rightarrow 0} \mathcal{B}_{\delta}(x) .
\end{gathered}
$$

Here we prove the following theorem.

THEOREM 2. If infinitely renormalizable maps $f$ and $g$ are both in the class $\mathcal{A}^{2}$, share the same bounded combinatorial type, and the conjugating homeomorphisms $h$ and $h^{-1}$ satisfy the Lipschitz condition on the postcritical Cantor sets $\mathcal{C}_{f}$ and $\mathcal{C}_{g}$ respectively, then $\mathcal{B}(x)=1$ at every $x \in \mathcal{C}_{f}$.

Proof. Similarly to the notions of $b(x)$ and $B(x)$ of the previous section $\mathcal{B}(x)$ is smoothly invariant and the chain rule can be applied. If there existed a point $x \in \mathcal{C}_{f}$ with $\mathcal{B}(x)=\gamma>1$ then since the action of a finite (though perhaps very long) composition of $f$ 's (or $g$ 's, respectively) is nearly linear on sufficiently small neighborhoods of $x$, and might be replaced by the composition of the derivatives, we could push this property forward to every arbitrarily small neighborhood of the critical point $c$. So it is enough to show that $\mathcal{B}(c)=1$. Suppose then that $\mathcal{B}(c)=\gamma>1$.

First we invoke two well known properties of maps in our class.

FACT 1. $\sum_{\Gamma \in C_{l}}|\Gamma|$ decreases exponentially in l for every infinitely renormalizable map (cf. $\S 3$ of [11]). 
FACT 2. The quantity

$$
\frac{\sum_{\Gamma \subset L_{n} \backslash L_{n+1}, \Gamma \in C_{l}}|\Gamma|}{\left|L_{n+1}\right|}
$$

is exponentially small in $l-n$ for infinitely renormalizable maps of bounded type $\leq T$, because of the "bounded geometry property" (cf. $\$ 15$ of [11]).

Now consider an interval $\Lambda \in C_{l}$ ( $l$ will vary in the considerations below) and another interval $\Omega \subset \Lambda, \Omega \in \bigcup C_{i}$ (we should think of $\Omega$ as being very short relative to $\Lambda$ or $\Omega \in C_{l+p}, p \gg 1$ ). Let $s$ be the first moment when the itinerary of $\Lambda$ under $f$ intersects $L_{n}, f^{s} \Lambda \subset L_{n}$. First we shall explain that the distortion of the ratio of the lengths $\Omega / \Lambda$ along this part of the itinerary is negligible, provided $l-n$ is large enough.

Consider the quantity

$$
\exp \left\{\left|\log \left(\frac{|\Lambda|}{\left|f^{s} \Lambda\right|}: \frac{|\Omega|}{\left|f^{s} \Omega\right|}\right)\right|\right\} .
$$

By Facts 1 and 2, for sufficiently large $l-n$ this quantity can be made arbitrarily close to 1 . This is true because in the part of the itinerary that goes through $I \backslash U$ (see the notation at the beginning of the previous section) $f^{\prime}$ is Lipschitz, bounded away from zero and we can use Fact 1 there; the total distortion coming from that part of the itinerary disappears exponentially fast in $l$. On the set $U, f^{\prime}$ can be small and we need the bounded geometry property there. If $l-n$ is large then the images of $\Lambda$ till time $s$ all have lengths very small relative to their distances to the critical point. By Fact 2, also the part of the itinerary that goes through $U$ contributes to the distortion of the quantity (11) from 1 only the amount that is a tail of a geometric series. So given $\varepsilon>0$, there exists $n_{0}$ such that if $l-n \geq n_{0}$, this tail sums up to less than $\varepsilon$. Then the logarithm of the quantity (11) is much smaller than $\log \gamma$. We can also claim the same for $g$, increasing $n_{0}$ if necessary.

Thus, if we had $|\Lambda| /|\bar{\Lambda}|=\tau$ and $|\Omega| /|\bar{\Omega}|=\sigma$ we can still have an approximate equality

$$
\frac{\left|f^{s} \Lambda\right|}{\left|g^{s} \bar{\Lambda}\right|}: \frac{\left|f^{s} \Omega\right|}{\left|g^{s} \bar{\Omega}\right|} \approx \frac{\tau}{\sigma}
$$

with an arbitrarily small error.

Above, we have been using the chain rule for the part of the itinerary of $\Lambda$ relatively distant from the critical point. Now we deal with those returns where the length of the image of $\Lambda$ is no longer negligible compared to its distance from $c$. There are only finitely many such steps - at most $n_{0}^{T}$, where $n_{0}$ was chosen to fit the required accuracy of an a priori picked $\varepsilon>0$. There we will use the existence of $h^{\prime}(c)$, already proven in Section 2 , as the "checkpoint" - relative positions of conjugate long intervals (i.e. those whose lengths are comparable to their distances from zero) are about the same 
for $f$ and $g$. For those return times we essentially use the homogeneity of $|x|^{\alpha}$ _-same relative positions are changed in the same way for both $f$ and $g$. It is also important that the lengths of the images of $\Omega$ are still negligible.

If the length of $\Lambda$ is comparable to its distance from $c$, but we are in a very small vicinity of $c$ (i.e. $n$ is very large), we can have the following approximate equalities:

$$
\frac{\left|f^{s} \Lambda\right|}{\left|g^{s} \bar{\Lambda}\right|} \approx h^{\prime}(c)
$$

and also

$$
\frac{\operatorname{dist}\left(c, f^{s}(\Omega)\right)}{\operatorname{dist}\left(\bar{c}, g_{s}(\bar{\Omega})\right)} \approx h^{\prime}(c)
$$

with an arbitrarily small error (recall we assumed $|\Omega| /|\Lambda| \ll 1$ ). In the set $U$, the maps $f$ and $g$ act as $|x|^{\alpha}$, so after one more step we have (again, with an arbitrarily small error)

$$
\frac{\left|f^{s+1} \Lambda\right|}{\left|g^{s+1} \bar{\Lambda}\right|} \approx \frac{\left|f^{s} \Lambda\right|}{\left|g^{s} \bar{\Lambda}\right|} \cdot \alpha \cdot\left(h^{\prime}(c)\right)^{\alpha-1}
$$

and if $\Omega$ was very short compared to $\operatorname{dist}(c, \Omega)$ we also have (with an arbitrarily small error)

$$
\frac{\left|f^{s+1} \Lambda\right|}{\left|g^{s+1} \bar{\Lambda}\right|}: \frac{\left|f^{s+1} \Omega\right|}{\left|g^{s+1} \bar{\Omega}\right|} \approx \frac{\tau}{\sigma} .
$$

Notice that we first choose and fix $n_{0}=n_{0}(\varepsilon)$ to make the change of $\tau / \sigma$ along the circuit far from the critical point very small and then we move that information toward the critical point by increasing $n$ to have the change of $\tau / \sigma$ arbitrarily small in the step close to the critical point. Now we go through the next circuit until the image of $f^{s+1} \Lambda$ falls again into $L_{n}$. Because the estimate of the change in $\tau / \sigma$ we gave above by Facts 1 and 2 was an upper estimate using all the intervals of $C_{l}$ not contained in $L_{n}$, we only used a fraction of those in the first circuit, and we can keep the same estimate for the itinerary containing the first and second circuits. Then we are again inside $L_{n}$ and use the argument about $|x|^{\alpha}$ a second time; then we follow the next circuit keeping the original estimate for the whole part of the itinerary outside $L_{n}$ and so forth till after no more than $n_{0}^{T}$ visits in $L_{n}, \Lambda$ falls onto $L_{l}$. But if $n$ was large enough (recall again we choose $n$ after $n_{0}$ was fixed), the change of $\tau / \sigma$ during the $n_{0}^{T}$ power-law steps can be made arbitrarily small. The conclusion of the above argument is the following property:

FACT 3. For any $\Lambda \in C_{l}$, and $\Omega \subset \Lambda, \Omega \in C_{l+p}, p>n_{0}$, if

$$
\frac{|\Lambda|}{|\bar{\Lambda}|}: \frac{|\Omega|}{|\bar{\Omega}|}=\frac{\tau}{\sigma}
$$


and $t$ is the smallest number such that $f^{t} \Lambda=L_{l}$ then

$$
\frac{\left|f^{t} \Lambda\right|}{\left|g^{t} \bar{\Lambda}\right|}: \frac{\left|f^{t} \Omega\right|}{\left|g^{t} \bar{\Omega}\right|} \approx \frac{\tau}{\sigma}
$$

with an arbitrarily small error, provided $l$ and $n_{0}$ are large enough.

Having established this, we proceed towards the completion of the proof. For any given interval $\Delta \in C_{n}$, we set

$$
\kappa(\Delta)=\inf _{\Gamma \subset \Delta, \Gamma \in \bigcup_{m \geq n} C_{m}} \frac{|\Gamma|}{|\bar{\Gamma}|},
$$

and analogously

$$
\mu(\Delta)=\sup _{\Gamma \subset \Delta, \Gamma \in \cup_{m \geq n} C_{m}} \frac{|\Gamma|}{|\bar{\Gamma}|} .
$$

Now we pick an interval $\Lambda \in C_{n}$, for some large $n$, such that $\mu(\Lambda) \approx \mathcal{M}$. Consider an arbitrary interval $\Omega \in \bigcup_{i \geq 0} C_{n+i}, \Omega \subset \Lambda$. We have the following dichotomy: either $\Omega \in \bigcup_{i=0}^{n_{0}} C_{n+i}$ (i.e. $\Omega$ is relatively long with respect to $\Lambda$ ), or $\Omega \in C_{l}$ with $l-n>n_{0}$. In the former case, because the derivative $h^{\prime}(c)$ exists, and $f^{t} \Lambda \ni c$ is very short, we have

$$
h^{\prime}(c) \approx \frac{\left|f^{t} \Omega\right|}{\left|g^{t} \bar{\Omega}\right|} .
$$

Here, as before, $t$ is the smallest number such that $f^{t} \Lambda$ is a basic renormalization interval containing $c$.

In the latter case, because of Fact 3 and by the definition of $\mathcal{M}$, we have an inequality

$$
\frac{\left|f^{t} \Omega\right|}{\left|g^{t} \bar{\Omega}\right|}<h^{\prime}(c)+\varepsilon
$$

where $\varepsilon$ can be arbitrarily small, provided $n_{0}, n$ were large enough. In either case, for all intervals $\Upsilon \in \bigcup C_{i}$ contained in a sufficiently small neighborhood of $c$, we have

$$
\frac{|\Upsilon|}{|\bar{\Upsilon}|}<h^{\prime}(c)+\varepsilon
$$

Now we pick another interval $\Lambda^{\prime} \in C_{n}$ such that $\kappa\left(\Lambda^{\prime}\right) \approx \mathcal{K}$. This time, because we start with the lower rather than the upper bound, the same argument shows that for all $\Upsilon \in \bigcup C_{i}$ in a sufficiently small neighborhood of $c$, the following inequality must hold true:

$$
\frac{|\Upsilon|}{|\bar{\Upsilon}|}>h^{\prime}(c)-\varepsilon
$$

But $\varepsilon$ could have been chosen arbitrarily small, for instance smaller than $(\gamma-1) / 4$, which contradicts $\mathcal{B}(c)>\gamma$. 
Considering the derivative $h^{\prime}(x)$ for $x \in \mathcal{C}_{f}$ (in the sense defined at the beginning of the previous section), the above theorem-by virtue of the Lipschitz condition - immediately yields the main result of this section:

COROLlary 1. If $f, g \in \mathcal{A}^{2}$ are infinitely renormalizable maps of the same bounded combinatorial type and the conjugating homeomorphisms $h$ and $h^{-1}$ satisfy the Lipschitz condition on the postcritical Cantor sets $\mathcal{C}_{f}$ and $\mathcal{C}_{g}$ respectively, then for every $x \in \mathcal{C}_{f}$ the first derivative $h^{\prime}(x)$ exists, and the derivative function $h^{\prime}$ is continuous in the metric on $\mathcal{C}_{f}$ inherited from $I$.

\section{References}

[1] V. I. Arnold, On the mapping of a circle into itself, Izv. Akad. Nauk SSSR Ser. Mat. 25 (1961), 21-86 (in Russian).

[2] P. Collet and J. P. Eckmann, Iterated Maps of the Interval as Dynamical Systems, Birkhäuser, Boston, 1980.

[3] A. Finzi, Problème de la génération d'une courbe fermée, Ann. Sci. École Norm. Sup. 67 (1950), 243-305.

[4] M. Herman, Sur la conjugaison différentiable des difféomorphismes du cercle à des rotations, Publ. Math. I.H.E.S. 49 (1979), 5-234.

[5] W. de Melo and S. van Strien, One-Dimensional Dynamics, Springer, Berlin, 1993.

[6] W. Pałuba, The Lipschitz condition for the conjugacies of Feigenbaum-like mappings, Fund. Math. 132 (1989), 227-258.

[7] -, A case of monotone ratio growth for quadratic-like mappings, Bull. Polish Acad. Sci. Math. 52 (2004), 381-393.

[8] D. Rand, Global phase space universality, smooth conjugacies and renormalization I. The $C^{1+\alpha}$ case, Nonlinearity 1 (1988), 181-202.

[9] W. Shen, Decay of geometry for unimodal maps: an elementary proof, in: Abstracts of ICM 2002 Satellite Conference, New Directions in Dynamical Systems, Kyoto 2002.

[10] M. Shub and D. Sullivan, Expanding endomorphisms of the circle revisited, Ergodic Theory Dynam. Systems 5 (1985), 285-289.

[11] D. Sullivan, Bounds, quadratic differentials and renormalization conjectures, in: AMS Centennial Publications, Vol. II (Providence, RI, 1988), Amer. Math. Soc., Providence, RI, 1992, 417-466.

[12] J.-C. Yoccoz, Conjugaison différentiable des difféomorphismes du cercle dont le nombre de rotation vérifie une condition Diophantienne, Ann. Sci. École Norm. Sup. 17 (1984), 333-361.

Institute of Mathematics

University of Warsaw

Banacha 2

02-097 Warszawa, Poland

E-mail:paluba@mimuw.edu.pl

Received 15 September 2003;

in revised form 6 September 2004 